\title{
RESPUESTA A LA INTERVENCIÓN Y ESCRITURA EN NIÑOS DE GRUPOS SOCIALES VULNERADOS
}

\author{
Response to intervention and spelling acquisition \\ in children from low-income backgrounds
}

https://doi.org/10.22235/pe.v10i2.1426

\author{
BEATRIZ DIUK*1 \\ MARINA FERRONI ${ }^{* 2}$ \\ MILAGROS MENA*3 \\ JUAN PABLO BARREYRO**4
}

*CONICET - Universidad Nacional de San Martín. Argentina. Correspondencia:

Recibido: 13/10/16

beadiuk@gmail.com; ferronimarina@gmail.com; mena_milagros@hotmail.com

Revisado: 25/11/16

**Universidad de Buenos Aires. Correspondencia: jpbarreyro@gmail.com

Aceptado: 09/05/17

Resumen: El objetivo del estudio fue analizar la respuesta a la intervención (RAI) en niños en contextos de pobreza con bajo nivel de alfabetización respecto de sus pares. Durante seis meses, 37 alumnos de 7 a 14 años de edad participaron de un programa de enseñanza individual. Los niños completaron una prueba de escritura de palabras al comenzar y al finalizar la intervención. Se obtuvieron diferencias significativas entre el pre y pos test. Un análisis de regresión sobre la ganancia en escritura mostró que la cantidad de sesiones del programa tuvo un efecto significativo. Solamente un $16 \%$ de los niños presentó baja RAI, lo que indica que las dificultades de la mayor parte de los niños serían experienciales y no resultado de déficit cognitivo.

Palabras clave: aprendizaje, escritura, enseñanza, pobreza, dificultades.

\begin{abstract}
The paper aimed to analyze the Response to Intervention (RTI) in children growing up in poverty contexts and exhibiting low levels of literacy when compared to their school peers. For six months, 37 7-to-14year-old children (41\% girls) participated in an educational program administered individually. Children were given a word spelling task before and after the intervention. Statistically significant differences were obtained between pre- and post-test. A regression analysis carried out on spelling gains showed that the number of program sessions children participated in had a significant effect. Only $16 \%$ of the children had a low RTI, indicating that most children's difficulties were experiential rather that related to cognitive deficits.
\end{abstract}

Keywords: learning, teaching, spelling, poverty, difficulties.

\footnotetext{
${ }^{1}$ Licenciada en Ciencias de la Educación y doctora en Psicología. Investigadora adjunta del CONICET. Docente y coordinadora del Centro de Investigaciones Psicopedagógicas Aplicadas de la Universidad Nacional de San Martín.

2 Licenciada en Letras y doctora en Psicología. Investigadora asistente del conicet, Argentina. Docente de la Universidad Nacional de San Martín, Argentina.

${ }^{3}$ Licenciada en Psicopedagogía. Becaria doctoral del conicet, Argentina. Docente de la Universidad Nacional de San Martín, Argentina.

4 Licenciado y doctor en Psicología. Investigador adjunto del conicet. Docente de la Facultad de Psicología, Universidad de Buenos Aires, Argentina.
} 


\section{INTRODUCCIÓN}

En el contexto de las sociedades latinoamericanas, caracterizadas por una profunda desigualdad (Comisión Económica para América Latina y el Caribe, 2015), los niños de sectores socioeconómicamente vulnerados suelen presentar mayores dificultades en las trayectorias escolares que niños de otros sectores sociales (Andrés, Canet-Juric, Richard's, Introzzi y Urquijo, 2010).

Una de las principales dificultades que enfrentan estos niños reside en su bajo nivel de alfabetización (Abadzi, 2008; Bizama, Arancibia y Sáez, 2013). En la actualidad, es posible encontrar en Argentina niños que cursan el último año de la escuela primaria sin poder leer ni escribir palabras sencillas, tal como evidencian los datos del presente estudio. Reiteradamente, la problemática de estos alumnos es interpretada como resultado de carencias de los propios niños (Apabalza, 2014; Ossa, Castro, Castañeda Díaz y Castro Rubilar, 2014), a pesar de los sostenidos planteos desarrollados desde perspectivas sociológicas y antropológicas acerca de la incidencia de las características del sistema educativo en la conformación de esta situación (Valencia, 2010).

En el marco de la atribución de las dificultades en la alfabetización a factores educacionales, y no a déficits de los propios niños, el presente trabajo se propone generar evidencia empírica acerca del potencial de los niños para aprender, a partir de incorporar a niños con muy bajo nivel de alfabetización a una propuesta de enseñanza sistemática (propuesta DALE!) y analizar sus niveles de respuesta a la intervención (RAI) pedagógica.

Las preguntas que orientan el estudio aquí reportado son: ¿es posible lograr, mediante una intervención sistemática aunque acotada en el tiempo, que aprendan a escribir niños que no lo han logrado a pesar de haber asistido al menos dos años a la escuela?, ¿es posible diferenciar entre niños con alto y bajo nivel de RAl?, ¿qué porcentaje de niños presentará un bajo nivel de respuesta?

\section{Dificultades de lectura y escritura en contextos socioeconómicamente vulnerados: explicaciones tradicionales y el modelo de la respuesta a la intervención}

Las explicaciones sobre las dificultades que los niños de sectores vulnerados enfrentan en su escolarización han tendido a polarizarse en torno a dos marcos interpretativos (Espíndola y León, 2002). Por un lado, se ha propuesto que los niños de los sectores más pobres carecerían de la preparación o de las capacidades necesarias para realizar el aprendizaje lector en los primeros años de escolarización. Se sostiene que no cuentan con la estimulación apropiada en el hogar, por lo que sus habilidades cognitivas serían inferiores a las de niños de otros sectores sociales (Kelly, 2012; Lus, 1995; Valencia, 2010). 
Desde una postura alternativa, los investigadores de la tradición sociocultural han sugerido que las dificultades que experimentan los niños más pobres resultan del desajuste entre los patrones de uso del lenguaje, y los modos de aprendizaje de estos y sus comunidades, y aquellos que la escuela espera y demanda (Dickinson y McCabe, 2001). Si bien este tipo de interpretación encuentra consenso en ámbitos académicos, el sistema escolar continúa operando en muchos casos con base en la atribución del fracaso en el aprendizaje a las competencias propias de los niños (Apabalza, 2014).

Tradicionalmente, los modelos y teorías elaborados en el campo de la psicología de la lectura no proporcionaban marcos que facilitaran la reconceptualización de las dificultades en el ámbito escolar. En efecto, predominaba en este campo una concepción de las dificultades que las explicaba estrictamente en términos de las habilidades y los conocimientos de los sujetos (Snow, Burns y Griffin, 1998).

Hace ya dos décadas, Vellutino y colaboradores (1996) cuestionaron este abordaje, al alegar que se produce una confusión entre niños con dificultades específicas de lectura y escritura - atribuibles a factores neurobiológicos- y niños con dificultades asociadas a la inadecuación de las prácticas de enseñanza. Así, desde mediados de la década de 1990, la concepción de las dificultades de lectura y escritura comenzó a modificarse, y se han desarrollado modelos que reconocen que el perfil cognitivo de todo sujeto es producto de la interacción entre factores de riesgo y factores protectores, tanto biológicos como ambientales (Bishop, 2006; Snowling, 2008).

El reconocimiento de que factores experienciales, y no solamente neurobiológicos, pueden configurar bajos niveles de adquisición lectora permite cuestionar las estrategias tradicionales de identificación de niños con trastornos lectores con base en evaluaciones psicométricas. Comienza entonces a desarrollarse el modelo de la respuesta a la intervención. Se trata de una aproximación destinada a identificar y atender a estudiantes que no logran avanzar en sus aprendizajes al ritmo de sus pares (Fuchs y Vaughn, 2012; Hughes y Dexter, 2011).

La implementación de los modelos de RAl abarca tres niveles. El nivel 1 lo constituye el aula de enseñanza común, en la que se identifica a aquellos niños cuyo avance resulta inferior al de sus compañeros. Estos niños participan entonces del nivel 2 , una intervención pedagógica individual o en pequeños grupos. Solamente aquellos niños que presentan un bajo nivel de respuesta a las intervenciones de nivel 2 pasan al nivel 3, que implica la derivación a especialistas (Fletcher y Vaughn, 2009).

El recurso a un modelo de la RAl puede contribuir a la comprensión de la etiología de las dificultades de los niños (Fletcher y Vaughn, 2009). De este modo, se distingue entre niños con un alto y un bajo nivel de RAl: Ios primeros serían niños cuyas dificultades se resuelven ante una intervención pedagógica adecuada y sistemática, mientras que los segundos serían aquellos cuyas dificultades persisten a pesar de la enseñanza sistemática y personalizada. Las dificultades iniciales experimentadas por niños que luego exhiben un alto nivel de respuesta serían fundamentalmente de origen experiencial, y es por ello que, ante una propuesta educativa adecuada, se resuelven. 
La persistencia de las dificultades en los niños con bajo nivel de RAI sería, por su parte, indicadora de dificultades de procesamiento más básicas: habría en estos niños alguna vulnerabilidad cognitiva que obstaculiza el aprendizaje (Greulich et al., 2014). De hecho, la psicología cognitiva actual considera que la persistencia de las dificultades lectoras constituye un potente indicador de una posible dislexia (Snowling, 2008). Si bien resulta debatible la idea de que todo niño con bajo nivel de RAl tiene un déficit de aprendizaje, ciertamente es posible afirmar que los niños con buen nivel de respuesta no lo padecen (Fletcher y Vaughn, 2009; Jiménez, 2010; Reynolds y Shaywitz, 2009).

El potencial de los modelos de RAl para distinguir entre niños que no aprenden por factores experienciales de niños con potenciales dificultades específicas para el aprendizaje cuenta en la actualidad con considerable evidencia empírica. Así, por ejemplo, Dougherty, Keane y Simic (2013) mostraron cómo dos instituciones que adoptaron el modelo de RAI redujeron notablemente el porcentaje de niños derivados para su evaluación por profesionales dada la presunción de la presencia de una dificultad específica para el aprendizaje. En una de las escuelas, el porcentaje de niños derivados descendió de $4,8 \%$ a $0,8 \%$ y en la otra, de 3,2 \% a 1,1\%. Este resultado fue producto del hecho de que las dificultades de niños que en años anteriores hubiesen sido derivados a especialistas pudieron ser resueltas mediante una intervención pedagógica sistemática en la escuela. En el mismo sentido VanDerHeyden, Witt y Gilbertson (2007) mostraron cómo, con la implementación de un modelo de RAl, no solamente el número de derivaciones se redujo, sino que se incrementó la confiabilidad de las derivaciones, que además reflejaron una representación sociocultural más equitativa. También Torgesen (2009) reportó una reducción en la proporción de niños derivados a educación especial.

De todos modos, estas investigaciones fueron desarrolladas en contextos muy distintos al latinoamericano, y las dificultades identificadas en los niños participantes de estos estudios difieren considerablemente de los niños incorporados en la presente investigación. En efecto, en los estudios anglosajones, se trata de niños con un nivel de precisión y de velocidad lectora relativamente reducidos. En el estudio actual, en cambio, se incluye una situación más compleja, dado que se trata de niños que, a pesar de haber pasado entre dos y cinco años en la escuela primaria, no han aprendido a escribir palabras. Aquí cobra relevancia un estudio realizado en Guatemala con niños de la etnia K'iche' (Rodas de Ruiz, 2014), en el que una intervención enmarcada en los modelos RAl dio lugar a un importante avance en escritura de palabras en los niños que comenzaron sin poder leer ni escribir.

En resumen, este trabajo analiza el nivel de RAI de niños crecidos en contextos de pobreza y que no habían aprendido a leer y a escribir, en el marco de una propuesta pedagógica destinada a atender a esta población específica (Propuesta DALE!, Diuk, 2013). 
Los niños participaron de la propuesta DALE!, y sus niveles de escritura de palabras fueron evaluados antes de incorporarse y nuevamente al finalizar el año escolar. Con el objeto de explorar la incidencia de variables potencialmente moduladoras del proceso de aprendizaje, se incluyó a niños con distintas edades, de diversos grados escolares y con diferente cantidad de sesiones de la propuesta DALE!

\section{MÉTODO}

\section{Participantes}

Participaron de este estudio 37 niños argentinos (15 niñas y 22 niños). La muestra forma parte de un proyecto de implementación de la propuesta DALE! en el conurbano de Buenos Aires; esta involucró a 100 niños y niñas, que fueron atendidos en forma individual, dos veces a la semana, por educadores especialmente capacitados en la propuesta. Se incorporó exclusivamente a niños que se encontraran en el nivel 1 de la propuesta al comenzar el año y que hubiesen participado de una cantidad mínima de sesiones (20) implementadas con un alto grado de fidelidad al programa original, según el criterio de las asesoras DALE! que visitaban cada experiencia. El nivel 1 incluye a niños que no pueden escribir en forma completa ninguna palabra no memorizada.

Los niños se incorporaron a la propuesta DALE! en sus escuelas (22 niños) o en centros de apoyo escolar (CAE) a los que concurrían a contraturno de la escuela (15 niños). Todos estaban escolarizados entre $2 .^{\circ}$ y $6 .^{\circ}$ grado de la escuela primaria. La incorporación de los niños al programa de intervención se produjo, inicialmente, a partir de su identificación por parte de los docentes que trabajaban con ellos en la escuela o en los CAE. Los niños eran identificados por no haber aprendido a leer y a escribir a pesar de presentar al menos dos años de escolarización. Posteriormente, se los evaluaba mediante una prueba de escritura de palabras a fin de corroborar su bajo nivel de desempeño y la necesidad de incorporarlos al programa.

No se incluyeron niños con trastornos sensoriales ni alteraciones del lenguaje ya diagnosticados. Todos los niños restantes que cumplieran con el requisito de no haber aprendido a leer y a escribir fueron incluidos, dado que, precisamente, la lógica de los modelos de RAI involucra una primera instancia de instrucción pedagógica para determinar qué niños presentan un bajo nivel de RAl y justifica, en consecuencia, una derivación para una evaluación específica.

Los niños tenían una edad promedio de 9,08 (DS $=1,8)$ con un rango entre los 7,7 y 14,5 años. El nivel socioeconómico de las familias fue ponderado según el nivel ocupacional de la madre o el padre de cada niño. La categorización de las ocupaciones se realizó con base en la escala ocupacional de Sautú (1992). En los casos en los que tanto la madre como el padre de un niño tuviesen trabajos rentados, se consideró a aquel que realizara el trabajo que puntuara más alto en esta escala. 
Todos los adultos de la muestra realizaban tareas correspondientes a los tres niveles más bajos de la escala, que cuenta con nueve categorías. La inclusión de los niños, tanto en el programa DALE! como en este trabajo, fue acordada con las familias, que firmaron un consentimiento informado.

\section{Instrumentos}

Se administró una prueba experimental de escritura de 36 palabras graduadas por nivel de dificultad en su estructura fonológica. En primer lugar, se incluyeron tres palabras muy familiares ("mamá", "oso", "casa"), que los niños suelen aprender a escribir a partir de la memorización del patrón ortográfico. Las siguientes palabras presentaban una estructura silábica simple, conformada por una consonante y una vocal. Las restantes palabras presentaban estructuras fonológicas más complejas, con sílabas de estructura CVC o CCV.

La administración de la prueba consistió en el dictado, por parte del entrevistador, de las palabras una por una. Las palabras se repetían tantas veces como los niños lo solicitaran, pero no se realizaban aclaraciones respecto de su escritura. La prueba se interrumpía si el niño no lograba escribir en forma completa 6 palabras.

La asignación de puntaje se realizó siguiendo un criterio fonológico, esto es, atendiendo a que cada fonema de la palabra estuviera representado por un grafema que pudiera representar a dicho fonema en algún contexto ortográfico, aunque no se tratara estrictamente del grafema convencional para la palabra dictada (por ejemplo, escribir "terasa" por "terraza" es considerado fonológicamente correcto, aunque no lo sea desde un punto de vista ortográficamente convencional).

\section{Procedimiento}

Tal como se señaló, los niños fueron identificados en primer lugar por sus docentes, y posteriormente se les administró la prueba de escritura de palabras en pequeños grupos. A continuación, participaron de la propuesta DALE! entre los meses de mayo y noviembre, en las instituciones educativas a las que concurrían.

La propuesta DALE! fue implementada por maestras tituladas, especialmente capacitadas en el marco teórico y metodológico de la propuesta. Cada maestra era visitada en forma quincenal por una asesora del equipo DALE!, quien garantizaba así la fidelidad de la implementación. La cantidad de sesiones de las que participó cada niño dependió de la asistencia de este a la escuela o el CAE, así como de factores organizativos de las instituciones, que facilitaron u obstaculizaron el desarrollo de la propuesta. En el mes de noviembre, se administró nuevamente la prueba de escritura de palabras, en este caso, de manera individual en un espacio tranquilo de las escuelas o los CAE. 


\section{La propuesta DALE!}

La propuesta DALE! —Derecho a Aprender a Leer y a Escribir- (Diuk, 2013) busca proporcionar una intervención pedagógica adaptada a las necesidades de los niños que no han aprendido al ritmo de sus pares. Ha sido desarrollada articulando una concepción sociocultural de los procesos de enseñanza y aprendizaje (Borzone, Rosemberg, Diuk, Silvestri y Plana, 2011), con los modelos y teorías sobre adquisición de la lectura y la escritura desarrollados en el marco de la psicología cognitiva en las últimas décadas (Feld, 2014; Vellutino, Fletcher, Snowling y Scanlon, 2004).

La propuesta contempla la asistencia de los niños dos veces a la semana, en sesiones de entre veinte minutos y media hora cada vez. La implementación está a cargo de un educador DALE! (que puede, o no, ser un docente titulado). Cada una de las sesiones presenta una organización estructurada en varios momentos, y en cada uno de ellos el docente a cargo cuenta con un importante repertorio de actividades que desarrolla según el nivel de conocimientos y el ritmo de avance de cada niño o niña.

Las sesiones de trabajo comienzan con una conversación sobre aspectos significativos de la vida del niño, de la que se desprende una oración o texto breve que el niño escribe con mucha ayuda del docente. A continuación, se desarrolla un trabajo sistemático con la escritura de palabras de distinto nivel de complejidad.

Este trabajo varía en función del nivel de conocimientos de los niños, y puede incluir desde el trabajo con las correspondencias hasta la adquisición de la ortografía. Se cierra la sesión con situaciones de lectura de palabras o textos. En el caso de los niños con menores conocimientos, la lectura está a cargo del docente, pero a medida que progresa el aprendizaje del niño, este asume cada vez mayores responsabilidades. ${ }^{5}$

\section{RESULTADOS}

En la Tabla 1 se presentan los puntajes obtenidos por los niños en la prueba de escritura al comenzar y al finalizar el año. Asimismo, se consignan los promedios de edad, grado escolar y cantidad de sesiones de las que participaron los niños.

\footnotetext{
5 Una presentación más extensa de la propuesta, así como los materiales para educadores y niños, pueden encontrarse en 〈http://www.propuestadale.com〉).
} 
Tabla 1. Promedios de edad, cantidad de sesiones y puntajes promedio en escritura de principio y fin de año

\begin{tabular}{lcccc}
\hline & $M$ & $D E$ & Mín. & Máx. \\
\hline Edad & 138.97 & 21.82 & 92 & 168 \\
Grado & 3.24 & 1.21 & 2 & 6 \\
Cantidad de sesiones & 20.49 & 10.07 & 3 & 40 \\
Prueba de escritura - Pre & 0.62 & 1.06 & 0 & 4 \\
Prueba de escritura - Post & 18.65 & 12.07 & 0 & 36 \\
\hline$N=37$. & & & & \\
\hline
\end{tabular}

Fuente: Elaboración propia (2015)

La inspección de las distribuciones de los puntajes obtenidos reveló que la tarea de escritura de palabras en el pre-test se alejó significativamente de la distribución normal asintótica ( $Z$ de Kolmogorov-Smirnov $=2.41, p=0.001$ ) por lo que en los análisis que incluyeron a esta prueba se utilizaron estadísticos no paramétricos. Mientras tanto, la prueba de escritura en el pos-test mostró una distribución que no se alejó significativamente de la distribución normal asintótica ( $Z$ de Kolmogorov-Smirnov = $0.82, p=0.52$ ).

Con el objeto de explorar si se produjeron diferencias significativas en el desempeño de los niños entre el comienzo y la finalización de su participación en la propuesta, se utilizó una prueba no paramétrica de contraste ( $U$ de Wilcoxon) para las medidas del pre y el pos-test. El análisis mostró diferencias estadísticamente significativas entre las pruebas $(Z=5.09, p=0.001)$. Este hecho revela que, efectivamente, se produjo un aprendizaje durante el período analizado. Si bien se trata de una prueba no estandarizada, debe tenerse en cuenta que, al comenzar, los niños escribieron, en promedio, menos de una palabra.

Es decir, a pesar de estar escolarizados y, en algunos casos, asistiendo a centros de apoyo escolar en forma complementaria a la escuela, en los años previos a la participación en el programa DALE!, no habían logrado aprender a leer y a escribir en forma autónoma ni siquiera palabras muy sencillas. Al finalizar su participación en el programa, escribieron correctamente, en promedio, 18 palabras. Este resultado por sí mismo aporta evidencia en el sentido de que, dadas las oportunidades educativas adecuadas, la mayor parte de los niños pudo aprender. 
Con el objeto de explorar la relación entre los conocimientos de escritura de los niños y variables tales como su edad, el grado al que asistían y la cantidad de sesiones de la propuesta DALE! de las que participaron, se calcularon las correlaciones por rangos de Spearman entre estas variables. Los resultados de este análisis mostraron que solamente se obtuvieron correlaciones significativas entre la cantidad de sesiones de las que los niños participaron y el desempeño en escritura al finalizar la intervención (ver Tabla 2). Resultaron no significativas las correlaciones entre la prueba del pre-test y la edad $(p=.786)$, el grado $(p=.472)$ y la cantidad de sesiones $(p=.759)$. La edad y el grado tampoco presentaron correlaciones significativas con la edad $(p=.139)$ y el grado $(p=.094)$.

Tabla 2. Correlaciones entre las medidas incluidas en el estudio

Prueba de escritura - Pre Prueba de escritura - Post

\begin{tabular}{lll}
\hline Edad & .05 & .25 \\
Grado & .12 & .28 \\
Cantidad de sesiones & .05 & $.57^{\star *}$ \\
\hline${ }^{* *} \mathrm{p}<.01$. & &
\end{tabular}

Fuente: Elaboración propia (2015)

Uno de los principales objetivos del presente estudio reside en explorar el nivel de RAI. En este sentido, se analizó a continuación el nivel de respuesta de los distintos niños, a fin de indagar en qué medida resultaba posible discriminar entre niños con alto y bajo nivel de RAl. Con este propósito, se calculó la ganancia en la intervención, restando el puntaje obtenido del pos-test sobre el pre-test. Esta puntuación obtenida, cuya media fue de $17.95(D E=11.98)$, mostró una distribución que no se diferenció significativamente de los percentiles teóricos de una distribución normal asintótica ( $Z$ de Kolmogorov-Smirnov $=0.78, p=0.59$ ). Este puntaje se puede definir como la ganancia obtenida por el niño en la prueba de escritura de palabras gracias a la intervención.

A continuación, se realizó un análisis de regresión lineal múltiple, tomando como variable dependiente al puntaje obtenido de la ganancia en la intervención y como variables independientes a la edad, el grado y la cantidad de sesiones DALE! EI modelo de regresión resultó significativo $F_{(3.33)}=8.94, M S E=86.41, p<0.001, R^{2}=0.40, y$ mostró que de todas las variables introducidas, solamente la cantidad de sesiones supo explicar la ganancia en la intervención $(\beta=.790, p<0.001)$, no así la edad ( $\beta=.54, p=$ $0.072)$ ni tampoco el grado $(\beta=-0.60, p<0.073)$. 
El modelo de regresión lineal múltiple permite ver el ajuste lineal de un conjunto de variables sobre una variable a predecir. En este caso, permite conocer el ajuste de la mejor combinación lineal de las variables independientes sobre la variable dependiente. Aunque es imposible graficar la recta en la dispersión de los valores, debido a que hay cuatro variables involucradas y no es posible realizar gráficos en cuatro dimensiones, sí se puede estimar este ajuste lineal matemáticamente. Esto es, se puede obtener el puntaje esperado para el chico en función de las variables independientes. Este puntaje esperado es la ganancia esperada a partir de la cantidad de sesiones. Gracias a ello, resulta posible estimar cuáles participantes no se ajustaron al puntaje esperado, que se obtiene al calcular la diferencia entre el puntaje obtenido por ellos y el puntaje esperado.

Los niños con bajo nivel de RAl son aquellos que se encuentran un desvío estándar por debajo en la diferencia entre el puntaje esperado y obtenido. En la muestra estudiada, seis niños fueron identificados con esta característica, quienes representan el $16.22 \%$ del total de niños de este estudio. En la figura 1 puede observarse el ajuste lineal de la relación entre el puntaje esperado y el puntaje obtenido por cada niño.

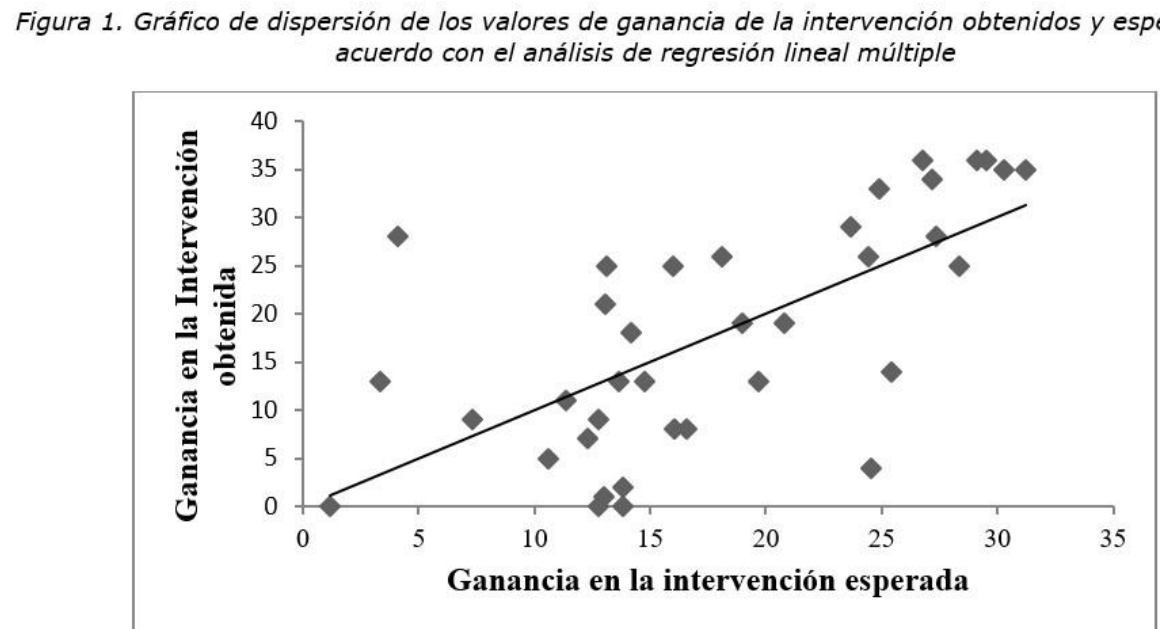

Fuente: Elaboración propia (2015) 


\section{DISCUSIÓN}

El presente trabajo se propuso explorar la RAl de un grupo de niños crecidos en contextos de pobreza y que, a pesar de llevar al menos dos años asistiendo a la escuela, no habían logrado aprender a escribir. Con frecuencia se considera que los niños que presentan estas situaciones carecen de las capacidades para el aprendizaje. Sin embargo, el supuesto que orientó este estudio fue que la mayor parte de los niños lograrían adquirir el sistema de escritura si se les proporcionaba una enseñanza adecuada, poniendo en evidencia así que sus dificultades no se relacionaban con problemas cognitivos propios, sino con factores relativos a las oportunidades para el aprendizaje. Para ello, los niños participaron de un programa de enseñanza de la lectura y la escritura (propuesta DALE!) destinado a la atención de alumnos que no avanzan al ritmo de sus pares.

En primer lugar, se analizó en qué medida resultaba posible que los niños avanzaran en su aprendizaje durante la intervención propuesta. Los resultados mostraron que, efectivamente, se produjo un aprendizaje en la mayor parte de los niños. Si se tiene en cuenta que se trata de niños que llevaban entre 2 y 5 años dentro del sistema educativo sin aprender a leer y escribir, los avances realizados resultan notables.

Cabe señalar que este aprendizaje estuvo asociado a la cantidad de sesiones del programa de las que cada niño participó. En efecto, el número de sesiones fue mejor predictor del desempeño al finalizar la intervención que la edad o el grado al que los niños concurrían en la escuela. Este resultado contribuye a validar la propuesta como un instrumento efectivo para promover el aprendizaje.

El número promedio de sesiones de las que participaron los niños fue 20 , con un máximo, en algunos casos, de 40 . Si se tiene en cuenta que cada sesión cuenta con una duración de 20 minutos, los resultados de este trabajo fueron alcanzados con un promedio de 7 horas de intervención pedagógica (y un máximo de 14 horas). En la literatura sobre modelos de RAl, se considera que un programa es intensivo si proporciona a los niños aproximadamente 100 horas de intervención complementaria a la del aula (Wanzek y Vaughn, 2009). Esto es, los niños lograron avanzar en sus niveles de aprendizaje aun con una intervención que puede ser considerada relativamente breve.

De todos modos, la mayor parte de las investigaciones reportadas en revistas científicas han sido desarrolladas con niños que aprendían a leer y a escribir en inglés, una lengua en la que el dominio del sistema de escritura es más complejo que en español (Share, 2008). La diferencia en tiempos de intervención entre la reportada aquí y la presentada en la bibliografía es de tal magnitud que resulta posible sostener que los niños respondieron a la enseñanza con una intervención de intensidad muy limitada. 
El hecho de que los niños del presente estudio hayan logrado avanzar en sus aprendizajes, a pesar de lo acotado del tiempo de enseñanza, aporta evidencia empírica al segundo de los objetivos del trabajo, consistente en explorar si resultaba posible diferenciar entre niños con alto y bajo nivel de la RAl.

Tal como se señaló en la introducción, el desarrollo de los modelos de RAI tuvo lugar a partir de un cambio de perspectiva en la psicología de la lectura, que implicó una ampliación del objeto de estudio para incorporar variables ambientales en el análisis de las dificultades lectoras. En este sentido, se considera que un buen nivel de RAl es indicador de que la problemática es primordialmente de origen experiencial (Schatschneider, Wagner y Crawford, 2008).

Ahora bien, a pesar de los buenos resultados generales, fue posible identificar a un grupo de niños de bajo nivel de RAI. Existe en la actualidad un considerable debate en torno a la identificación de los malos respondedores (Tolar, Barth, Fletcher, Francis y Vaughn, 2014), dado que distintas metodologías dan lugar a la identificación de niños diferentes (Toste et al., 2014). En este trabajo se tomó la decisión de identificar a los malos respondedores respecto del propio grupo de niños que participó de la intervención pedagógica. Hace ya varios años, un equipo de destacados investigadores en el área advirtió acerca de las limitaciones de la estrategia de identificar a los niños con dificultades respecto de muestras estandarizadas y sugirió utilizar a los propios grupos de pertenencia como medida de comparación (Snow et al., 1998).

Otro aspecto crítico en la identificación de los niños con baja RAl refiere a la arbitrariedad del punto de corte. En este estudio se optó por utilizar una medida relativamente conservadora, de 1 desvío respecto de la media, con lo que el porcentaje de niños con bajo nivel de RAl fue de $16 \%$. Si se utilizara como punto de corte 1,5 desvíos, solamente dos niños calificarían como malos respondedores $(5 \%$ de la muestra original). El porcentaje de malos respondedores varía mucho en función de los estudios. Al Otaiba y Fuchs (2002) reportaron que en distintos estudios los porcentajes oscilaron entre el 8 y el $80 \%$. El $16 \%$ obtenido es relativamente bajo respecto del $25 \%$ más frecuente en estudios recientes (Toste et al., 2014). Este bajo porcentaje remite, nuevamente, a la naturaleza predominantemente experiencial de la dificultad de los niños.

Una de las limitaciones de este estudio fue el hecho de no contar con un grupo de comparación. Esta opción se debió a motivaciones éticas. Incorporar un grupo de comparación habría involucrado negar oportunidades de aprendizaje a niños que realmente las necesitaban, solamente a los fines de la investigación. Si bien esta práctica es ocasionalmente identificada en el campo (Wanzek y Vaughn, 2008), los autores de este trabajo, así como los equipos educativos a cargo de la propuesta, consideraron que no resultaba aceptable

Los resultados alcanzados tienen importantes implicancias pedagógicas. Por un lado, ponen en evidencia que, con una intervención pedagógica sistemática y basada en la evidencia internacional acerca de las mejores estrategias de enseñanza, resulta 
posible promover la adquisición del sistema de escritura en niños que aparentemente tendrían importantes dificultades para lograrlo. Es precisamente esta evidencia, combinada con el reducido porcentaje de niños con bajo nivel de RAl, la que puede aportar a profundizar el debate acerca del origen experiencial (educativo en particular) de las dificultades que enfrentan numerosos niños argentinos que crecen en contextos de pobreza. En este sentido, se vuelve fundamental promover en el sistema educativo la generación de condiciones que efectivamente hagan posible el aprendizaje de todos los niños (Ossa et al., 2014).

El estudio presenta la limitación de contar con un tamaño muestral restringido. Este hecho se relaciona con la complejidad que implica sostener una intervención pedagógica en instituciones en contextos de pobreza extrema que además cumpla con requisitos de cantidad de sesiones y fidelidad de la implementación. Actualmente, se está trabajando en la replicación de este estudio con una muestra más amplia.

\section{REFERENCIAS}

Abadzi, H. (2008). Aprendizaje eficaz y pobreza: Ideas desde las fronteras de la neurociencia cognitiva. Santiago, Chile: Ediciones Universidad Católica Silva Henríquez.

Al Otaiba, S., y Fuchs, D. (2002). Characteristics of children who are unresponsive to early literacy intervention: A review of the literature. Remedial and Special Education, 23, 300-316. doi:10.1177/07419325020230050501

Andrés, M. L., Canet Juric, L., Richard's, M., Introzzi, I., y Urquijo, S. (2010). Disponibilidad de recursos materiales en el hogar y adquisición de habilidades pre-lectoras. Psicologia Escolar e Educacional, 14(1), 139-148.

Apabalza, M. (2014). Representaciones sociales de profesores respecto de la diversidad escolar en relación a los contextos de desempeño profesional, prácticas y formación inicial. Estudios Pedagógicos, 40(1), 7-24.

Bishop, D. (2006). Developmental cognitive genetics: How psychology can inform genetics and vice versa. The Quarterly Journal of Experimental Psychology, 59, 1153-1168. doi: 10.1080/17470210500489372

Bizama, M., Arancibia, B., y Sáez, K. (2013). Intervención psicopedagógica temprana en conciencia fonológica como proceso metalingüístico a la base de la lectura en niños de 5 a 6 años socialmente vulnerables. Estudios Pedagógicos, 39(2), 25-39.

Borzone, A. M., Rosemberg, C. R., Diuk, B., Silvestri, A., y Plana, D. (2011). Niños y maestros por el camino de la alfabetización. Buenos Aires, Argentina: Novedades Educativas.

Comisión Económica para América Latina y el Caribe. (2015). América Latina y el Caribe: una mirada al futuro desde los Objetivos de Desarrollo del Milenio. Informe regional de monitoreo de los Objetivos de Desarrollo del Milenio (ODM) en América Latina y el Caribe. Recuperado de http://repositorio.cepal.org/bitstream/handle/11362/38923/S1500709 es.pdf?sequence $=5$ 
Dickinson, D. K., y McCabe, A. (2001). Bringing it all together: The multiple origins, skills and environmental supports of early literacy. Learning Disabilities Research y Practice, 16(4), 186-202. doi: 10.1111/0938-8982.00019

Diuk, B. (2013). Propuesta DALE! Guía para el Docente. Buenos Aires, Argentina: FPC y Etis.

Dougherty, K., Keane, A., y Simic, O. (2013). Translating policy to practice: initiating RTI in urban schools. Urban Education, 48, 350-379.

Espíndola, E., y León, A. (2002). La deserción escolar en América Latina: Un tema prioritario para la agenda regional. Revista Iberoamericana de Educación, 30, 39-62.

Feld, V. (2014). Las habilidades fonológicas, su organización neurofisiológica y su aplicación en la educación. Pensamiento Psicológico, 12(1), 71-82.

Fletcher, J. M., y Vaughn, S. (2009). Response to intervention: Preventing and remediating academic difficulties. Child Development Perspectives, 3(1), 30-37. doi: 10.1111/j.1750-8606.2008.00072.x.

Fuchs, L. S., y Vaughn, S. (2012). Responsiveness-to-intervention a decade later. Journal of Learning Disabilities, 45(3), 195-203. doi: 10.1177/0022219412442150

Greulich, L., Al Otaiba, S., Schatschneider, C., Wanzek, J., Ortiz, M., y Wagner, R. (2014). Understanding Inadequate Response to First-Grade Multi-Tier Intervention: Nomothetic and Ideographic Perspectives. Learning Disability Quarterly, 37(4), 204-217. doi: 10.1177/0731948714526999

Hughes, C., y Dexter, D. (2011). Response to intervention: A research-based summary. Theory into Practice, 50(1), 4-11. doi: 10.1080/00405841.2011.534909

Jiménez, J. (2010). Response to Intervention (Rtl) Model: A promising alternative for identifying students with learning disabilities?. Psicothema, 22, 932-934.

Kelly, C. (2012). Recognizing the "social" in literacy as a social practice: Building on the resources of nonmainstream students. Journal of Adolescent y Adult Literacy, 55(7), 608-618.

Lus, M. A. (1995). De la integración escolar a la Escuela integradora. Buenos Aires, Argentina: Paidós.

Ossa, C., Castro, F., Castañeda Díaz, M., y Castro Rubilar, J. (2014). Cultura y liderazgo escolar: factores claves para el desarrollo de la inclusión educativa. Actualidades Investigativas en Educación, 14, 1-23.

Reynolds, C., y Shaywitz, S. (2009). Response to Intervention: Ready or Not? Or, From Wait-to-Fail to Watch-Them-Fail. School Psychology Quarterly, 24(2), 130-145. doi:10.1037/a0016158.

Rodas de Ruiz, P. (2014). Un acercamiento inicial al modelo RTI para estudiantes de un grupo lingüístico minoritario con necesidades en lectoescritura. Interamerican Journal of Psychology, 48, 194-202.

Sautú, R. (1992). Teoría y medición del estatus ocupacional. Escalas ocupacionales objetivas y de prestigio. Buenos Aires, Argentina: Universidad de Buenos Aires.

Schatschneider, C. L., Wagner, R. K., y Crawford, E. (2008). The importance of measuring growth in response to intervention models: Testing a core assumption. Learning and Individual Differences, 18, 308-315. doi: 10.1016/j.lindif.2008.04.005 
Share, D. L. (2008). Orthographic learning, phonology and the self-teaching hypothesis. Advances in Child Development and Behavior, 36, 31-82. doi:10.1016/S0065-2407(08)00002-5

Snow, C. E., Burns, M. S., y Griffin, P. (1998). Preventing reading difficulties in young children: Committee on the prevention of reading difficulties in young children. Washington, DC: National Research Council.

Snowling, M. (2008). Specific disorders and broader phenotypes: The case of dyslexia. The Quarterly Journal of Experimental Psychology, 61(1), 142-156. doi: 10.1080/17470210701508830

Tolar, T., Barth, A., Fletcher, J., Francis, D., y Vaughn, S. (2014). Predicting reading outcomes with progress monitoring slopes among middle grade students. Learning and Individual Differences, 30, 46-57. doi: 10.1016/j.lindif.2013.11.001

Torgesen, J. K. (2009). The response to intervention instructional model: Some outcomes from a large-scale implementation in Reading First schools. Child Development Perspectives, 3, 38-40.

Toste, J., Compton, D., Fuchs, D., Fuchs, L., Gilbert, J., Cho, C.,... Bouton, B. (2014). Understanding unresponsiveness to tier 2 reading intervention: exploring the classification and profiles of adequate and inadequate responders in first grade. Learning Disability Quarterly, 37, 192-203. doi: $10.1177 / 0731948713518336$

Valencia, R. (2010). Dismantling contemporary deficit thinking: Educational thought and practice. New York, NY: Taylor and Francis.

VanDerHeyden, A. M., Witt, J. C., y Gilbertson, D. (2007). A multi-year evaluation of the effects of a response to intervention (RTI) model on identification of children for special education. Journal of School Psychology, 45, 225-256.

Vellutino, F. R., Fletcher, J. M., Snowling, M. J., y Scanlon, D. M. (2004). Specific reading disability (dyslexia): What have we learned in the past four decades? Journal of Child Psychology and Psychiatry, 45(1), 2-40. doi:10.1046/j.0021-9630.2003.00305.x

Vellutino, F. R., Scanlon, D. M, Sipay, E., Small, S., Pratt, A., Chen, R., y Denckla, M. (1996). Cognitive profiles of difficult-to-remediate and readily remediated poor readers: Early intervention as a vehicle for distinguishing between cognitive and experiential deficits as basic causes of specific reading disability. Journal of Educational Psychology, 88, 601-638. doi: 10.1037/0022-0663.88.4.601

Wanzek, J., y Vaughn, S. (2008). Response to varying amounts of time in reading intervention for students with low response to intervention. Journal of Learning Disabilities, 41, 126-142. doi: $10.1177 / 0022219407313426$

Wanzek, J., y Vaughn, S. (2009). Students demonstrating persistent low response to reading intervention: Three case studies. Learning Disabilities: Research and Practice, 24, 151-163. doi: 10.1111/j.1540-5826.2009.00289.x 